\title{
An Efficient Fitness Function in Genetic Algorithm Classifier for Landuse Recognition on Satellite Images
}

\author{
Ming-Der Yang, ${ }^{1}$ Yeh-Fen Yang, ${ }^{1}$ Tung-Ching Su, ${ }^{2}$ and Kai-Siang Huang ${ }^{1}$ \\ ${ }^{1}$ Department of Civil Engineering, National Chung Hsing University, Taichung 40227, Taiwan \\ ${ }^{2}$ Department of Civil Engineering and Engineering Management, National Quemoy University, Kinmen 89250, Taiwan \\ Correspondence should be addressed to Yeh-Fen Yang; yf.yang@yahoo.com.tw
}

Received 30 August 2013; Accepted 4 December 2013; Published 18 February 2014

Academic Editors: J. Shu and F. Yu

Copyright (C) 2014 Ming-Der Yang et al. This is an open access article distributed under the Creative Commons Attribution License, which permits unrestricted use, distribution, and reproduction in any medium, provided the original work is properly cited.

Genetic algorithm (GA) is designed to search the optimal solution via weeding out the worse gene strings based on a fitness function. GA had demonstrated effectiveness in solving the problems of unsupervised image classification, one of the optimization problems in a large domain. Many indices or hybrid algorithms as a fitness function in a GA classifier are built to improve the classification accuracy. This paper proposes a new index, DBFCMI, by integrating two common indices, DBI and FCMI, in a GA classifier to improve the accuracy and robustness of classification. For the purpose of testing and verifying DBFCMI, well-known indices such as DBI, FCMI, and PASI are employed as well for comparison. A SPOT-5 satellite image in a partial watershed of Shihmen reservoir is adopted as the examined material for landuse classification. As a result, DBFCMI acquires higher overall accuracy and robustness than the rest indices in unsupervised classification.

\section{Introduction}

Novel techniques of image classification, including supervised and unsupervised classifications, have been developed and widely applied to the problems of pattern recognition. Supervised classification requires prior knowledge for the training of the classification model. Taking satellite image classification, for example, the prior knowledge, means the average and standard deviation of spectrum of each landuse. Such a prior knowledge has been taken as criteria and then the examined image is classified to the distinct object of interest referring to the criteria [1-5].

On the contrast, unsupervised classification can be implemented automatically by analyst-defined clustering criteria as the basis for classification rather than the training data set collected beforehand. Unsupervised classification groups a set of test data in such a way that the data within a class (cluster) are more similar in some identities to one another than in other groups. Unsupervised classification starts with a specific number of classes either arbitrarily in accordance with the research objectives or based on the analyst's expertise and then interprets all pixels within a data set into a correspondent class pixel by pixel. In accordance with such a merit, unsupervised classification is more suitable for the interpretation of environment with fragmentary land cover for areas or the image detection without prior statistics of the training data from the study field [6]. However, due to lacking of the ground truth, the accuracy of unsupervised classification is inferior to supervised classification [7]. Therefore, the accuracy improvement of unsupervised classification remains a critical issue needing a great effort.

Inspired by the nature evolution process, GA has been extensively and successfully applied to many practical problems, such as urban landscape change analysis [8], urban sprawl detection [9], multicomponent image segmentation [10], and image edge detection [11]. Therefore, how to apply GA to get better results has become a remarkable and practical topic during the past decade. GA can efficiently improve the results analyzed based upon heuristic methods away from the local solutions and then get the optimal results especially in image analysis and interpretation and artificial intelligence [12-14]. With this superiority, many GA researches were undertaken and developed. 
Bandyopadhyay and Maulik [15] integrated DaviesBouldin index, Dunn's index, Fuzzy C-means index, and Cindex into GA as fitness functions for clustering analysis. Bandyopadhyay and Maulik [16] also integrated K-means into clustering GA for unsupervised clustering to improve the defect of K-means needing the initial cluster numbers a prior and getting better results. Yang and Wu [17] established partition separation index and verified its superiority by comparing it with other five noted clustering indices: partition coefficient index, partition entropy index, Fukuyama and Sugeno validity function, Xie and Beni validity function, and Davies and Bouldin validity function.

Accordingly, GA operations can start the evaluation by individuals (so-called GA strings or chromosomes) of population (initial generation) being substituted over a specified number of generations which are consisted of the strings from one initial individual that swapped some segments between two strings (so-called crossover), so as to find the optimal fitness piece by piece [18]. Particularly, instead of searching the optimal solution from a few assigned points within the searching space of the training data, GAs can initialize a group of the solution sets selected randomly and automatically from the solution space [18]. This research developed a new index by including the merits of DBI and FCMI, so-called DBFCMI, to promote the accuracy of GAs. Normally, DBI considers both the distribution of inner cluster and between clusters with membership usually defined as classical crisp logic. However, while the interaction of pixels within an image is considered, the crisp membership function of classical logic seems unsuitable for the relationship of these pixels. Instead, FCMI based on fuzzy C-means (FCM) that is extended from a method known as hard C-means is adopted in a crisp classifying application, developed by Bezdek [19], and is an extremely powerful classification method for fuzzy data. However, FCMI considers only the dispersion of inner cluster without the dispersion between clusters; therefore, the clustering efficiency could cause the beneath clustering or the excessive clustering.

In this research, the new index was verified for its feasibility and stability via various initial sets (i.e., different lengths of chromosome and numbers of populations), selection ways, and crossover ways.

\section{Methodology}

2.1. GA Operator. GA, based on mimicking the natural strategies of evolution, can preserve the fittest which is one of the useful optimization techniques. A genetic string, so-called an individual, is encoded of a particular solution to a problem. And the solution must be able to express characteristics of the sample space. Before an operation of GA, a number of individuals are produced for the population of initial generation. Each genetic string is usually encoded by the types of binary, integer, or real number. After the operations of crossover and mutation, the possible solutions within the solution space are obtained and calculated their fitness according to a fitness function. Repeating the operations of evolution and preserving the fittest by selection, the possible solutions could be evaluated generation by generation until the optimal solution is derived.

2.1.1. GA Operating Steps. A genetic string is the foundation for establishing a genetic algorithm and could describe a possible solution to a problem. It is made of units what can represent the characters of the problem. An individual is a bit string of arbitrary units. Basically, the meaningful string length must consist of at least two and upper genes $[6,17]$. In this research, the string length is set in 8 and encoded with the integer number because of the radiometric resolution of SPOT-5 image and then each unit of the gene string is comprised of 4 units because of the 4 bands of the image. The member of a generation, so-called population, also influences clustering accuracy. Besides, referring to Coley [20], Liu et al. [21], and Sivanandam and Deepa [22], the member of a generation, so-called population, 30 through 90, is adopted in this research.

Once the initial generation is randomly selected from the universal set, some strings, even number usually, with superior fitness are partially selected into the crossover pool. Afterwards, new members of population based on the operations of crossover and mutation were generated for the next generation $[22,23]$. Roulette wheel selection and rank selection are the two common selection techniques that were adopted in this research.

Two typical parameters, including crossover probability $\left(P_{c}\right)$ and crossover way, must be determined. The crossover probabilities of 0.4 through 0.9 are usually suggested [20], so $P_{c}$ of 0.8 was adopted in this research. Besides, the most common popular crossover ways, such as single-point crossover, multipoint crossover, and uniform crossover, were adopted. In addition, three other crossover forms, including threeparent crossover, ordered crossover, and shuffle crossover, were also tested.

The purpose of mutation is to prevent GA from being trapped into local optimal solutions. A low mutation probability, typically between 0.001 and 0.01 , is given because a high mutation probability would change GA to random search. Sivanandam and Deepa [22] proposed that an appropriate mutation probability should be determined according to the reciprocal of string length. That is, supposing the length of the genetic string consists of 8 genes, the mutation probability is given as $1 / 8$. Flipping mutation has been widely applied to the enlarging solution spaces [22] so to be employed in this paper.

2.1.2. Fitness Function. In each generation, the priority of the genetic strings is ranked according to the fitness values calculated based on a fitness function. Through either maximizing or minimizing the fitness values generation by generation, the genetic string with the global optimum could be found to be the terminal clustering result.

Currently, many indices, such as K-means index (KMI), separation index (SI), partition separation index (PASI), Davies-Bouldin index (DBI), and fuzzy C-means index (FCMI), have been presented to be the fitness functions of GA. Among the previous indices, DBI considers the inner differences within a cluster as well as the differences among 


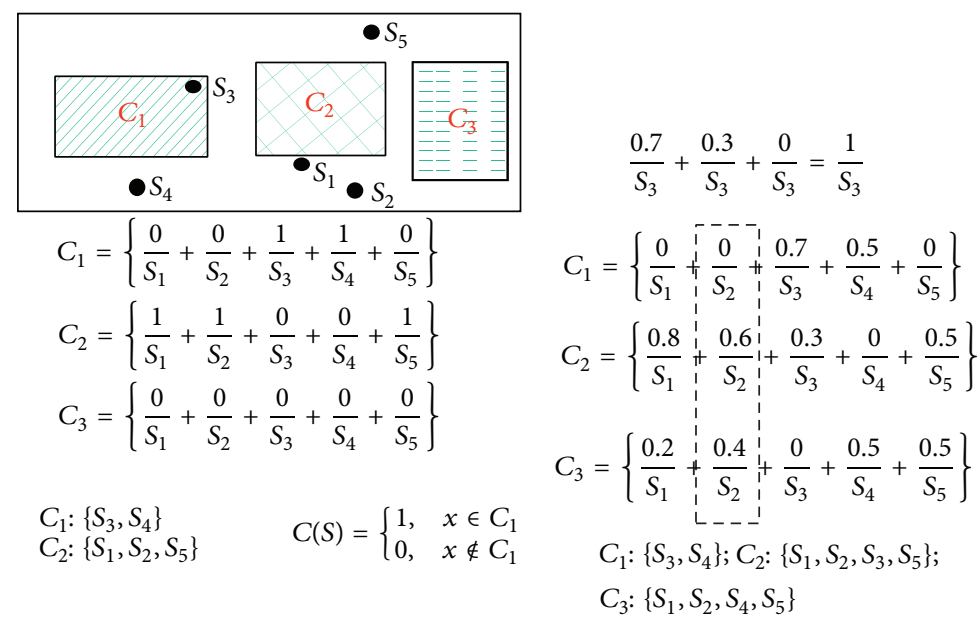

(a) Classic set

(b) Fuzzy set

FIGURE 1: The dissimilarity between classic set and fuzzy set.

the clusters so that the better clustering results could be acquired. However, rather than considering the influence between the other clusters, the specified pixel DBI considers only the influence between the specified pixel and the cluster it belonged to. FCM basically integrated fuzzy membership function with C-means clustering and then further integrating into GA as a fitness function, so-called FCMI, can be a complementary to DBI. Therefore, in this paper, DBFCMI, integrated FCMI with DBI, is built to attempt to obtain the better clustering accuracy.

Dunn [24] developed the fuzzy C-means (FCM) which had been successfully improved and applied to the clustering analysis by Bezdek [19]. The membership function of the fuzzy C-means is used to scale the weights of a data to the clustering centers into a continuous interval $[0,1]$ rather than the classical set with the crisp binary units 0 and 1 (see Figure 1). In Figure 1, the $C$ values express the clustering centers and the $x=\left\{x_{1}, x_{2}, x_{3}, \ldots, x_{n}\right\}$ denotes an analyzed image. The subscript $\mathrm{n}$ means the number of the pixels of an image.

Unlike DBI, FCMI considers the influence between each pixel and all cluster centers. That is, the distance between a pixel and the pixels in the same cluster will be considerably less than the distance between a pixel and the pixels in different clusters. Of course the reciprocal influence of the former one is considered larger than the latter one. Also the membership grade is considered based on the same distance measurement. The objective function of FCMI is shown as (1), and the optimal cluster centers can be found by minimizing (1). The center of the $j$ th cluster is determined by (2). Equation (3) is the membership function of $x_{i}$ being assigned to the $j$ th cluster. As for DBI, it can be obtained by the derivation of $(5)[3,15,19]$. The optimal cluster centers could be derived by maximizing DBI.

In order to demonstrate the performance of DBFCMI, this research referred to the literature of Yang and $\mathrm{Wu}$ [17] to apply several indices on a certain man-made data which display the different shapes of distribution. The experimental result indicates that the three indices, including DaviesBouldin index (DBI), partition separation index (PASI), and fuzzy C-means index (FCMI), have the better performances. Therefore, this research introduced the above three indices and DBFCMI into GA for the unsupervised clustering analysis.

DBFCMI is mainly based on DBI. Furthermore, it evaluates distance between a pixel and the cluster centers based on fuzzy membership rather than the distance between the pixel and the cluster center which the pixel belonged to (see (7)). The membership function was evaluated via (8).

(a) Fuzzy C-means index (FCMI)

$$
\begin{gathered}
\text { Min FCMI }=\sum_{j=1}^{c} \sum_{i=1}^{n} \mu_{j i}^{2}\left\|x_{i}-v_{j}\right\|^{2}, \\
v_{j}=\frac{\sum_{i=1}^{n} \mu_{j i}^{2} x_{i}}{\sum_{j=1}^{n} \mu_{j i}^{2}}, \quad j=1, \ldots, c, i=1, \ldots, n, \\
\mu_{j i}=\mu_{j}\left(x_{i}\right)=\left(\sum_{k=1}^{c} \frac{\left\|x_{i}-v_{j}\right\|^{2}}{\left\|x_{i}-v_{k}\right\|^{2}}\right)^{-1}, \\
\sum_{j=1}^{c} \sum_{i=1}^{n} \mu_{j i}(x)=1 .
\end{gathered}
$$

(b) Davies-Bouldin index (DBI)

$$
\begin{gathered}
\mu_{k n}= \begin{cases}1 ; & \left\|x_{n}-v_{k}\right\| \leq\left\|x_{n}-v_{j}\right\|, \\
0 ; & \text { otherwise }\end{cases} \\
1 \leq k, \quad j \leq K ; \quad j \neq k ; \quad 1 \leq n \leq N, \\
v_{k}=\frac{\sum_{n=1}^{M}\left(\mu_{k n}\right) x_{n}}{\sum_{n=1}^{M}\left(\mu_{k n}\right)}=\frac{\sum_{x_{n} \in X_{k}} x_{n}}{M_{k}}, \quad 1 \leq k \leq K,
\end{gathered}
$$




$$
\begin{gathered}
S_{j}=\left(\frac{1}{\left|X_{j}\right|} \sum_{x \subset X_{j}}\left\|x-v_{j}\right\|^{2}\right)^{1 / 2}, \\
S_{k}=\left(\frac{1}{\left|X_{k}\right|} \sum_{x \subset X_{k}}\left\|x-v_{k}\right\|^{2}\right)^{1 / 2}, \\
d_{k j, t}=\left\|v_{k}-v_{j}\right\|_{t}, \\
R_{k, t}=\max _{j \neq k}\left\{\frac{S_{k}+S_{j}}{d_{k j, t}}\right\}, \quad j=1, \ldots,(K-1) ; j \neq k, \\
\mathrm{DB}_{i}=\frac{1}{K} \sum_{k=1}^{K} R_{k, t}, \quad i=1, \ldots, m, \\
\operatorname{Max} \mathrm{DBI}=\frac{1}{\mathrm{DB}} .
\end{gathered}
$$

(c) Partition separation index (PASI)

$$
\begin{gathered}
\mathrm{PS}_{j}=\sum_{i=1}^{n} \frac{\mu_{j i}^{2}}{\mu_{M}}-\exp \left(\frac{-\min _{k \neq j}\left\{\left\|v_{j}-v_{k}\right\|^{2}\right\}}{\beta_{T}}\right), \\
\mu_{M}=\max _{1 \leq j \leq c}\left\{\sum_{i=1}^{n} \mu_{j i}^{2}\right\}, \quad \beta_{T}=\frac{\sum_{j=1}^{c_{j}}\left\|v_{j}-\bar{v}\right\|^{2}}{c_{j}}, \\
\operatorname{Max} \operatorname{PSI}(c)=\sum_{j=1}^{c} \operatorname{PS}_{j} .
\end{gathered}
$$

(d) Davies-Bouldin and fuzzy C-means index (DBFCMI)

$$
\begin{gathered}
v_{k}=\frac{\sum_{n=1}^{M}\left(\mu_{k n}\right) x_{n}}{\sum_{n=1}^{M}\left(\mu_{k n}\right)}=\frac{\sum_{x_{n} \in X_{k}} x_{n}}{M_{k}}, \quad 1 \leq k \leq K, \\
\mu_{j i}=\mu_{j}\left(x_{i}\right)=\left(\sum_{k=1}^{c} \frac{\left\|x_{i}-v_{j}\right\|^{2}}{\left\|x_{i}-v_{k}\right\|^{2}}\right)^{-1}, \\
v_{j}=\frac{\sum_{i=1}^{n} \mu_{j i}^{2} x_{i}}{\sum_{j=1}^{n} \mu_{j i}^{2}}, \quad j=1, \ldots, c, i=1, \ldots, n, \\
\sum_{j=1}^{c} \sum_{i=1}^{n} \mu_{j i}(x)=1, \\
S_{k}=\left(\frac{\sum_{j=1}^{c} \sum_{i=1}^{n} \mu_{j i}^{2}\left\|x_{i}-v_{i}\right\|^{2}}{N}\right)^{1 / 2}, \\
d_{k j, t}=\left\|v_{k}-v_{j}\right\|_{t} \\
R_{k, t}=\max _{j, j \neq k}\left\{\frac{S_{k}+S_{j}}{d_{k j, t}}\right\}
\end{gathered}
$$

TABLE 1: Spectral centers of landuse.

\begin{tabular}{lcccc}
\hline \multirow{2}{*}{ Landuse } & \multicolumn{4}{c}{ Band } \\
& B1 (NIR) & B2 (G) & B3 (R) & B4 (SWIR) \\
\hline Vegetation & 134.5 & 99.5 & 72.0 & 66.9 \\
Water & 58.8 & 52.4 & 55.9 & 57.1 \\
Forest & 112.1 & 98.3 & 57.3 & 58.4 \\
Bare land & 128.9 & 93.9 & 72.9 & 65.9 \\
Structure & 133.2 & 88.1 & 90.5 & 77.3 \\
\hline
\end{tabular}

$$
\begin{gathered}
\operatorname{DBFCM}_{i}=\frac{1}{K} \sum_{k=1}^{K} R_{k, t}, \quad i=1, \ldots, m, \\
\operatorname{Max} \operatorname{DBFCMI}=\frac{1}{\operatorname{DBFCM}_{i}} .
\end{gathered}
$$

2.1.3. Termination Criteria. There are two termination criteria for the GA operation, including the convergence of optimal solution searching or the specified number of generations that have evolved. Even though the latter termination criterion is adopted by most researchers $[3,10,12,21]$, it is too time-consuming for mega data, such as GA image classification. In this research, therefore, the convergence of optimal solution searching is set as the termination criterion for the GA operation.

2.2. Introduction of Study Site and SPOT-5. The study site is a hillside within the watershed of Shihmen reservoir located in Northern Taiwan (see Figure 2). The reservoir supplies water to 28 districts of Northern Taiwan, including 3.4 million people. The Shihmen reservoir serves a number of purposes, including irrigation, hydroelectric power, water supply, flood prevention, and sightseeing. Thus, the watershed and water monitoring of Shihmen reservoir become a very important job [25]. The economic activity of humanity in the watershed of Shihmen reservoir is an important factor that could influence the water quality. The landuse classification using remote sensing data can offer the administrator an efficient and real-time monitoring of the natural change and agricultural activities [26-28]. Landuse classification can offer the administrator an efficient and real-time monitoring for the economic activities of humanity. A SPOT-5 satellite image, which was photographed on August 19, 2006, was acquired as the experimental material. SPOT-5 satellite image has a panchromatic mode $(0.48 \mathrm{~m}-0.71 \mathrm{~m})$ with a spatial resolution of $5 \mathrm{~m}$ and a multispectral mode $(0.50 \mathrm{~m}-0.59 \mathrm{~m}$ in green, $0.61 \mathrm{~m}-0.68 \mathrm{~m}$ in red, $0.78 \mathrm{~m}-0.89 \mathrm{~m}$ in near IR, and $1.58 \mathrm{~m}-$ $1.75 \mathrm{~m}$ in short wave IR) with a spatial resolution of $10 \mathrm{~m}$. The size of the subset satellite image is $181 \times 171$ pixels (a total of 30,951 pixels). The ground truth data was produced from an aerial photography taken on August 14, 2006. The landuse patterns include vegetation, water, forest, bare land, and structure, whose spectral centers and standard deviations are listed in Tables 1 and 2. 


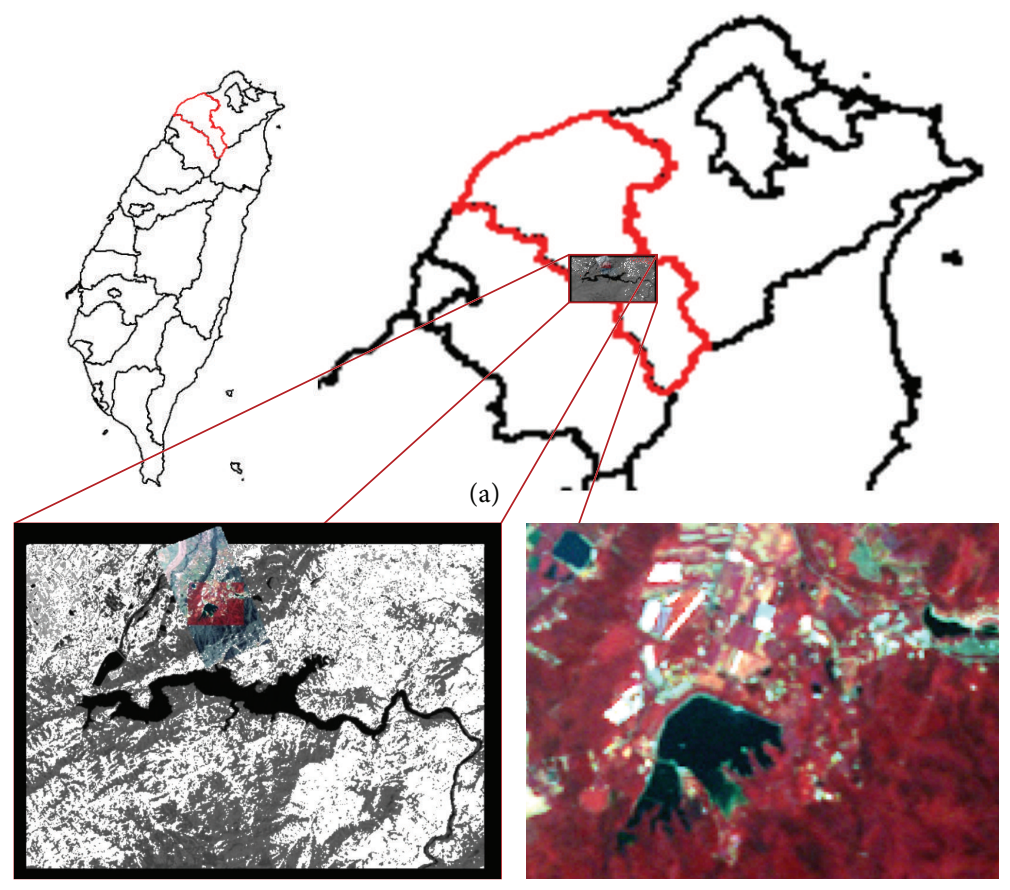

(b)

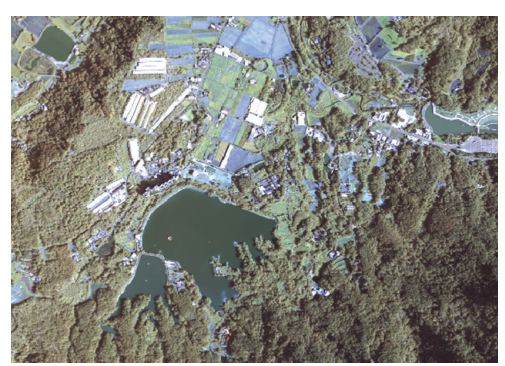

(d) (c)
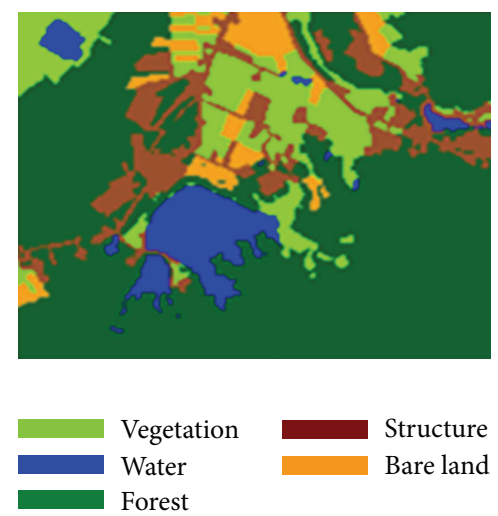

(e)

Figure 2: (a) Location of the Shihmen reservoir; (b) location of the study site; (c) the subset satellite image of the study site; (d) subset aerial photograph with the same studied range; (e) distributions of ground truth.

TABLE 2: Standard deviations of the spectrum.

\begin{tabular}{|c|c|c|c|c|c|c|}
\hline \multirow{3}{*}{ Landuse } & \multicolumn{6}{|c|}{ Band } \\
\hline & \multicolumn{4}{|c|}{ Standard deviation } & \multirow{2}{*}{$\begin{array}{c}\text { Overall } \\
\text { standard deviation }\end{array}$} & \multirow{2}{*}{$\begin{array}{l}\text { Threshold } \\
\qquad(2 \sigma)\end{array}$} \\
\hline & B1 & B2 & B3 & B4 & & \\
\hline Vegetation & 27.2 & 22.1 & 7.2 & 4.0 & 27.2 & 22.1 \\
\hline Water & 13.5 & 12.8 & 11.5 & 6.6 & 13.5 & 12.8 \\
\hline Forest & 13.4 & 11.2 & 7.4 & 4.2 & 13.4 & 11.2 \\
\hline Bare land & 17.7 & 14.4 & 12.4 & 5.5 & 17.7 & 14.4 \\
\hline Structure & 27.3 & 16.6 & 32.3 & 22.1 & 27.3 & 16.6 \\
\hline
\end{tabular}

Most indices whenever are integrated into GA might probably cause the excessive classifying. Therefore, expect the 5 categories of landuse in accordance with the surface; the other categories determined here by GA are all defined as the 6th landuse, so-called others.

\section{The Results}

3.1. The Results Varying with Populations. We implemented different GA operations settings in order to verify the stable optimum of DBFCMI. In this research, the different populations consisting of 30,60,75, and 90 string numbers coupled with the given GA parameters, including maximal string length of 8 genes [29], roulette wheel selection, twopoint crossover $[24,30]$, crossover probability of 0.8 , and mutation probability of $0.003[24,31]$, were tested. Tables 3 and 4 show the overall accuracy and K-HAT values derived from the stability analysis varying with the different fitness 
TABLE 3: Overall accuracy of each index varying with populations.

\begin{tabular}{lccccc}
\hline \multirow{2}{*}{ Population } & \multicolumn{5}{c}{ Index } \\
& DBI & FCMI & PASI & DBFCMI & Average \\
\hline 30 & 71.6 & 73.2 & 64.9 & 75.5 & 71.3 \\
60 & 74.7 & 69.3 & 68.6 & 72.9 & 71.4 \\
75 & 70.3 & 73.7 & 70.8 & 72.1 & 71.7 \\
90 & 73.8 & 65.7 & 70.7 & 73.9 & 71.0 \\
Standard & 2.0 & 3.7 & 2.8 & 1.4 & - \\
deviation & & & & & \\
\hline
\end{tabular}

${ }^{*}$ String length: 8 , selection way: roulette wheel selection, crossover rate: 0.8 , crossover way: two-point crossover, and mutation rate: 0.003 .

TABLE 4: K-HAT of each index varying with populations.

\begin{tabular}{lccccc}
\hline \multirow{2}{*}{ Population } & \multicolumn{5}{c}{ Index } \\
& DBI & FCMI & PASI & DBFCMI & Average \\
\hline 30 & 0.36 & 0.34 & 0.24 & 0.48 & 0.34 \\
60 & 0.35 & 0.24 & 0.07 & 0.33 & 0.25 \\
75 & 0.37 & 0.31 & 0.29 & 0.39 & 0.34 \\
90 & 0.32 & 0.21 & 0.16 & 0.36 & 0.26 \\
Standard & 0.02 & 0.06 & 0.10 & 0.04 & - \\
deviation & & & & & - \\
\hline
\end{tabular}

* String length: 8 , selection way: roulette wheel selection, crossover rate: 0.8 , crossover way: two-point crossover, and mutation rate: 0.003 .

indices. In the tables, DBFCMI can mostly lead to the better overall accuracy and K-HAT than the other indices, while the population is 60 . In Tables 5 and 6 , the best overall accuracy of $75.5 \%$ and the best K-HAT of 0.48 were derived, while the population is assigned to 30 based on DBFCMI.

The image classification results corresponding to overall accuracy and K-HAT values in Tables 3 and 4 are shown in Figure 3. Figure 3 shows that there were only 3 classes detected based on the best overall accuracy and K-HAT of $75.4 \%$ and $0.48 \%$. It is remarkable that DBI mostly can get the number of classifications as many as the ground truth. However, the pixels would mostly be assigned in incorrect classes especially in vegetation. Unlike DBFCMI, although the numbers of classifications are mostly under the number of real landuses, nevertheless, the distribution of each landuse is more corresponding to the ground truth. In Figure 3, it is remarkable that DBFCMI can distinguish structure from the other landuses more accurately and the distribution of structure is more corresponding to ground truth than the other associated models.

3.2. The Results Varying with Selection Ways. Optimal solution by elite selection in GA operations includes many ways. Two of them are adopted widely, that is, roulette wheel selection and rank selection. Thus, the two selection ways applied to the four indices were evaluated based on overall accuracy and K-HAT as well. The testing results presented in Tables 5 and 6 demonstrate that the DBFCMI can get higher
TABLE 5: Overall accuracy of each index varying with selection ways.

\begin{tabular}{lccccc}
\hline \multirow{2}{*}{ Selection way } & \multicolumn{5}{c}{ Index } \\
& DBI & FCMI & PASI & DBFCMI & Average \\
\hline $\begin{array}{l}\text { Roulette wheel } \\
\text { selection }\end{array}$ & 71.6 & 73.2 & 64.9 & 75.5 & 71.3 \\
$\begin{array}{l}\text { Rank selection } \\
\begin{array}{l}\text { Standard } \\
\text { deviation }\end{array}\end{array}$ & 68.3 & 47.7 & 69.1 & 75.1 & 65.0 \\
\hline
\end{tabular}

${ }^{*}$ String length: 8 , population: 30 , crossover rate: 0.8 , crossover way: twopoint crossover, and mutation rate: 0.003 .

TABLE 6: K-HAT of each index varying with selection ways.

\begin{tabular}{lccccc}
\hline & \multicolumn{5}{c}{ Index } \\
Selection way & \multicolumn{5}{c}{ K-HAT } \\
& DBI & FCMI & PASI & DBFCMI & Average \\
\hline $\begin{array}{l}\text { Roulette wheel } \\
\text { Selection }\end{array}$ & 0.36 & 0.34 & 0.24 & 0.48 & 0.34 \\
$\begin{array}{l}\text { Rank selection } \\
\begin{array}{l}\text { Standard } \\
\text { deviation }\end{array}\end{array}$ & 0.19 & 0.06 & 0.10 & 0.39 & 0.19 \\
* & 0.12 & 0.20 & 0.10 & 0.02 & - \\
$\begin{array}{l}\text { String length: 8, population: 30, crossover rate: 0.8, crossover way: two- } \\
\text { point crossover, mutation rate: } 0.003 .\end{array}$
\end{tabular}

values in both selection ways and roulette wheel selection outperforms rank selection for most indices except PASI.

Figure 4 shows the classified results varying with selection ways related to different indices. Comparing the same six classes between DBI (see Figure 3; population size is equal to 60) and DBFCMI, DBFCMI can distinguish more pixels of bare land than DBI properly and thus can get higher values of both overall accuracy and K-HAT. The results are used once again to verify the accuracy and stability of DBFCMI.

3.3. The Results Varying with Crossover Ways. In the way of crossover approaches, including single-point crossover (P1), two-point crossover (P2), multipoint crossover (P3), threeparent crossover (P4), ordered crossover (P5), and shuffle crossover (P6), related to the different indices, they were also tested in this research (see Tables 7 and 8) for the accuracy and stability analysis. Obviously, the better overall accuracy and K-HAT values could be derived from DBFCMI than the other indices as well as the advanced GA operations.

Figure 5 is the classified images related to Tables 7 and 8. Among these results from the indices varying with the different crossover ways, DBFCMI still can get the higher values of overall accuracy and K-HAT in substance except a K-HAT value of FCMI with the multipoint crossover way (see Table 8). Also, DBFCMI presents its power of distinguishing structure from the other landuses again although the classes are mostly beneath the number of ground truth. Besides, according to Figures 5 and 6 , it is presented that while DBI is integrated with the single-point crossover way (P1) and two-point crossover way (P2), the results will have the higher potential in excessive classifying than the other indices. 


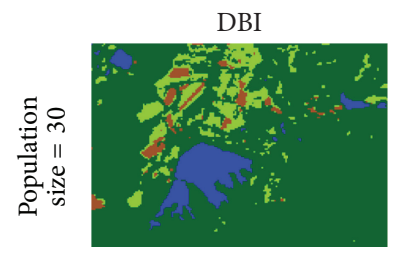

4 classes

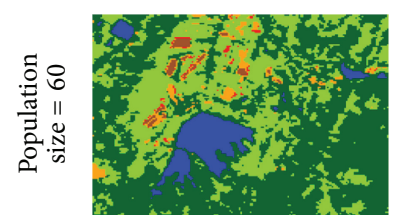

6 classes

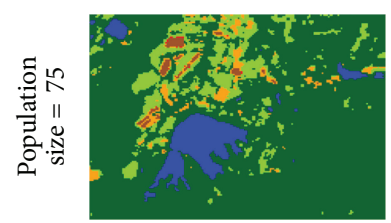

5 classes

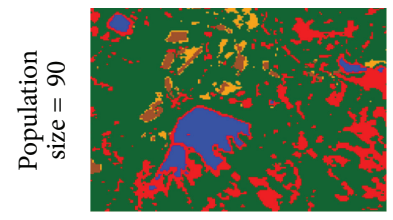

5 classes

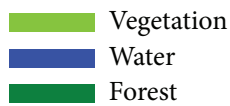

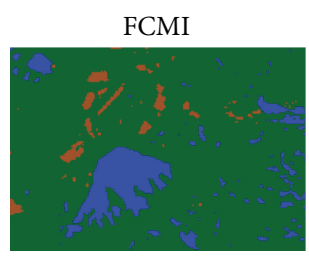

3 classes

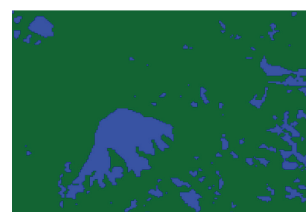

2 classes

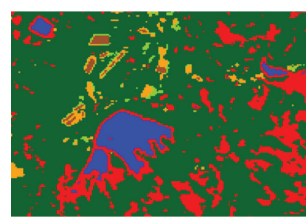

6 classes

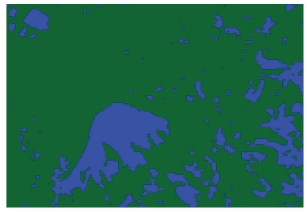

2 classes

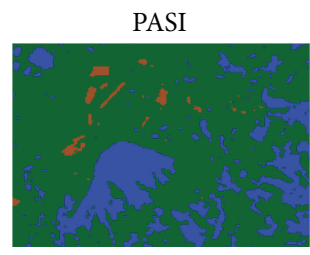

3 classes

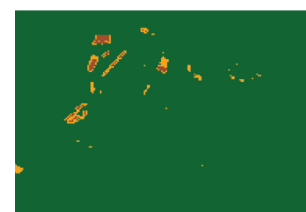

3 classes

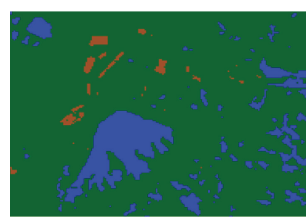

3 classes

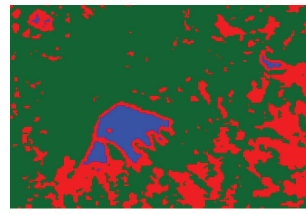

3 classes

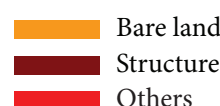

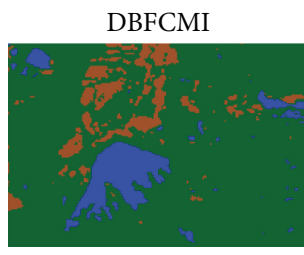

3 classes

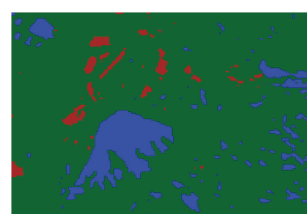

3 classes

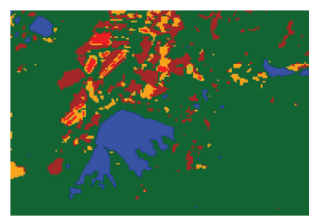

5 classes

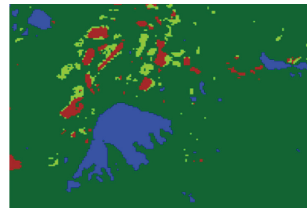

4 classes

FIGURE 3: The best result interpreted by the different indices varying with populations.

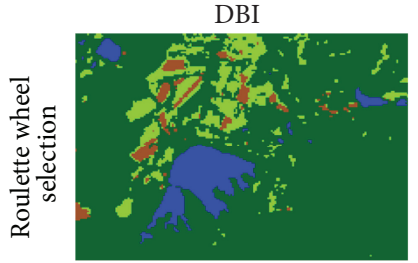

4 classes

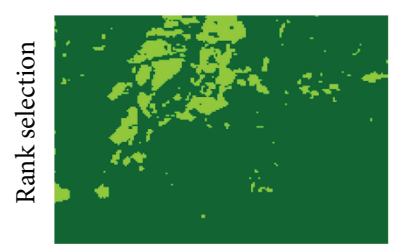

2 classes

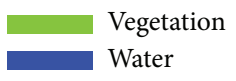

Water

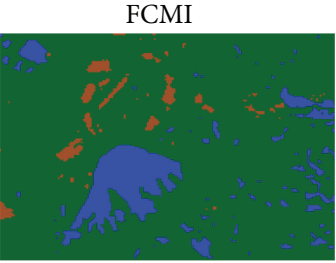

3 classes

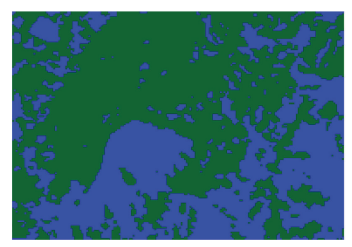

2 classes

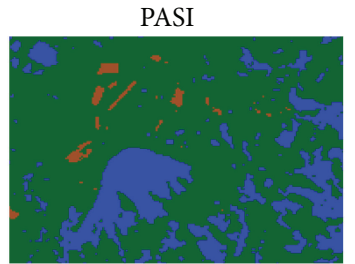

3 classes

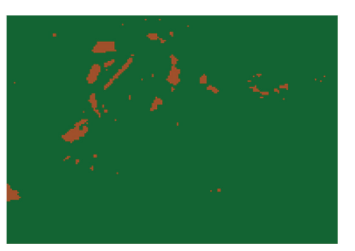

2 classes
DBFCMI

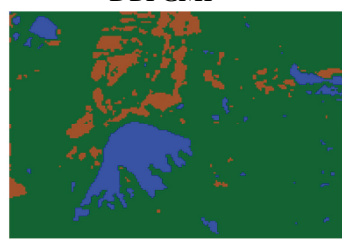

3 classes

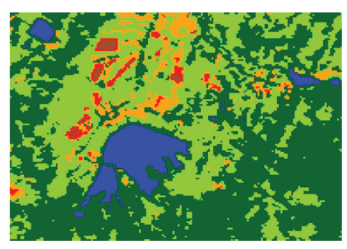

6 classes

FIGURE 4: The best result interpreted by the different indices varying with selection ways. 

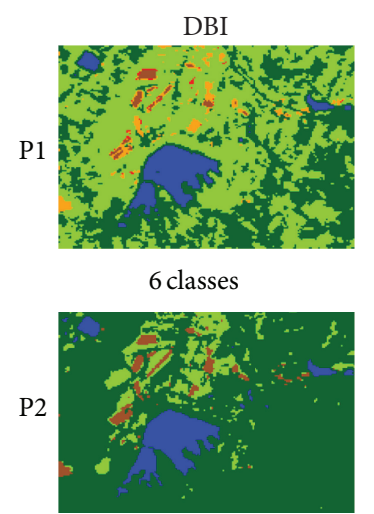

4 classes

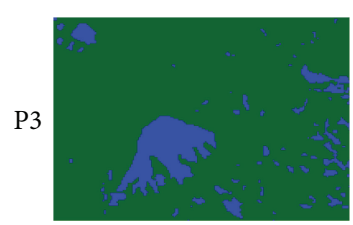

2 classes

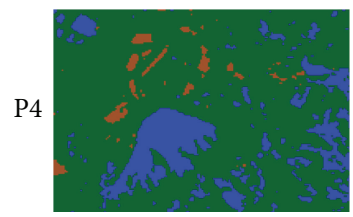

3 classes

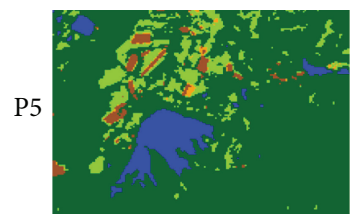

5 classes

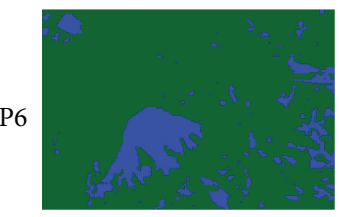

2 classes

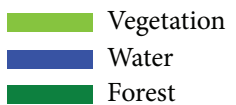

FCMI

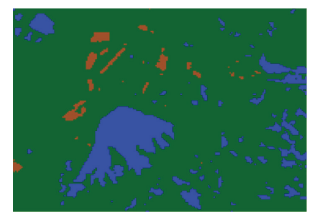

3 classes

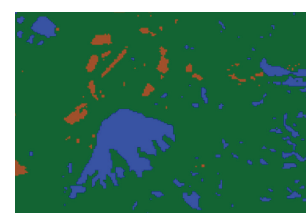

3 classes

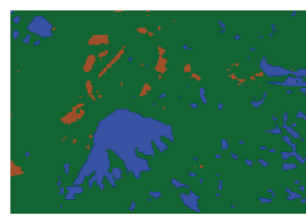

3 classes

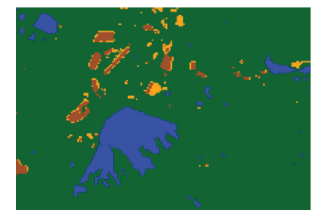

4 classes

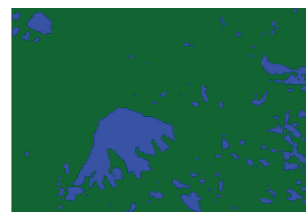

2 classes

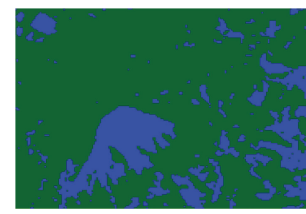

2 classes

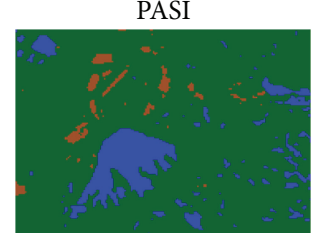

3 classes

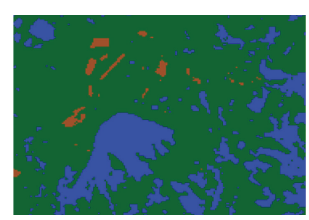

3 classes

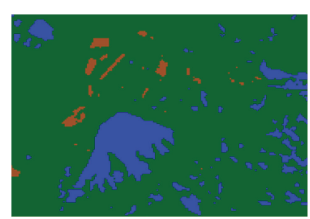

3 classes

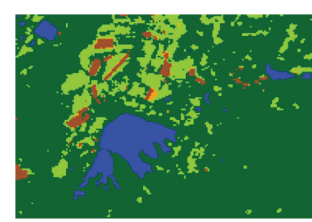

5 classes

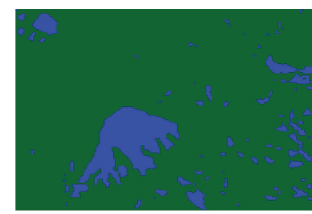

2 classes

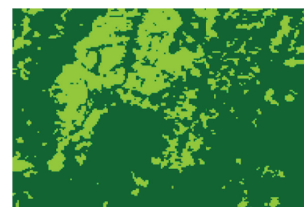

2 classes

Bare land

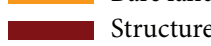

Others

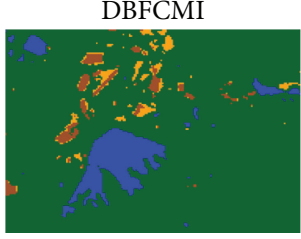

4 classes

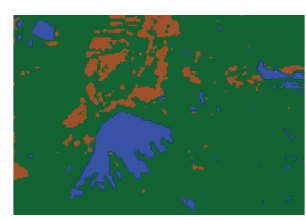

3 classes

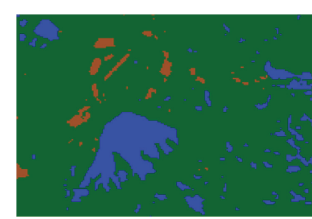

3 classes

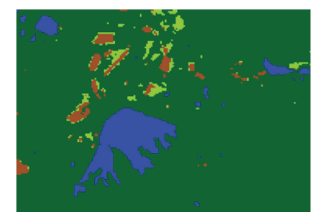

4 classes

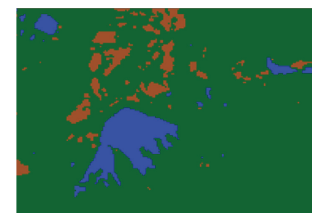

3 classes

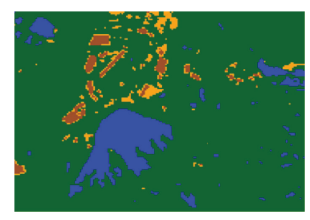

4 classes

FIGURE 5: The best result interpreted by the different indices varying with crossover ways.

\section{Results Analysis}

Figure 6 shows the spectral centrals of the optimal solutions based on the four indices varying with different GA operations versus ground truth. In the figure, the difference of spectral centers between the optimal solutions and ground truth can be observed evidently. Among the five landuses, bare land cannot be determined by all of the four indices with their optimal models. Even though bare land can be distinguished from the other landuses with some of the GA operations, the distribution of it principally disagrees with ground truth. On the contrary, among the optimal solutions, the centers of forest can be determined much closer to the ground truth in both classified centers and distribution than the other landuses due to its wide spectrum variation, and then vegetation, structure, and water are in sequence. DBFCMI can only determine three kinds of landuse (i.e., forest, water, and structure), however the spectral centers of the three landuses classified by DBFCMI seem to be closer to the ground truth than the other indices especially in forest and water. Among the GA classifications with four indices, FCMI results in the greatest difference between the classified 

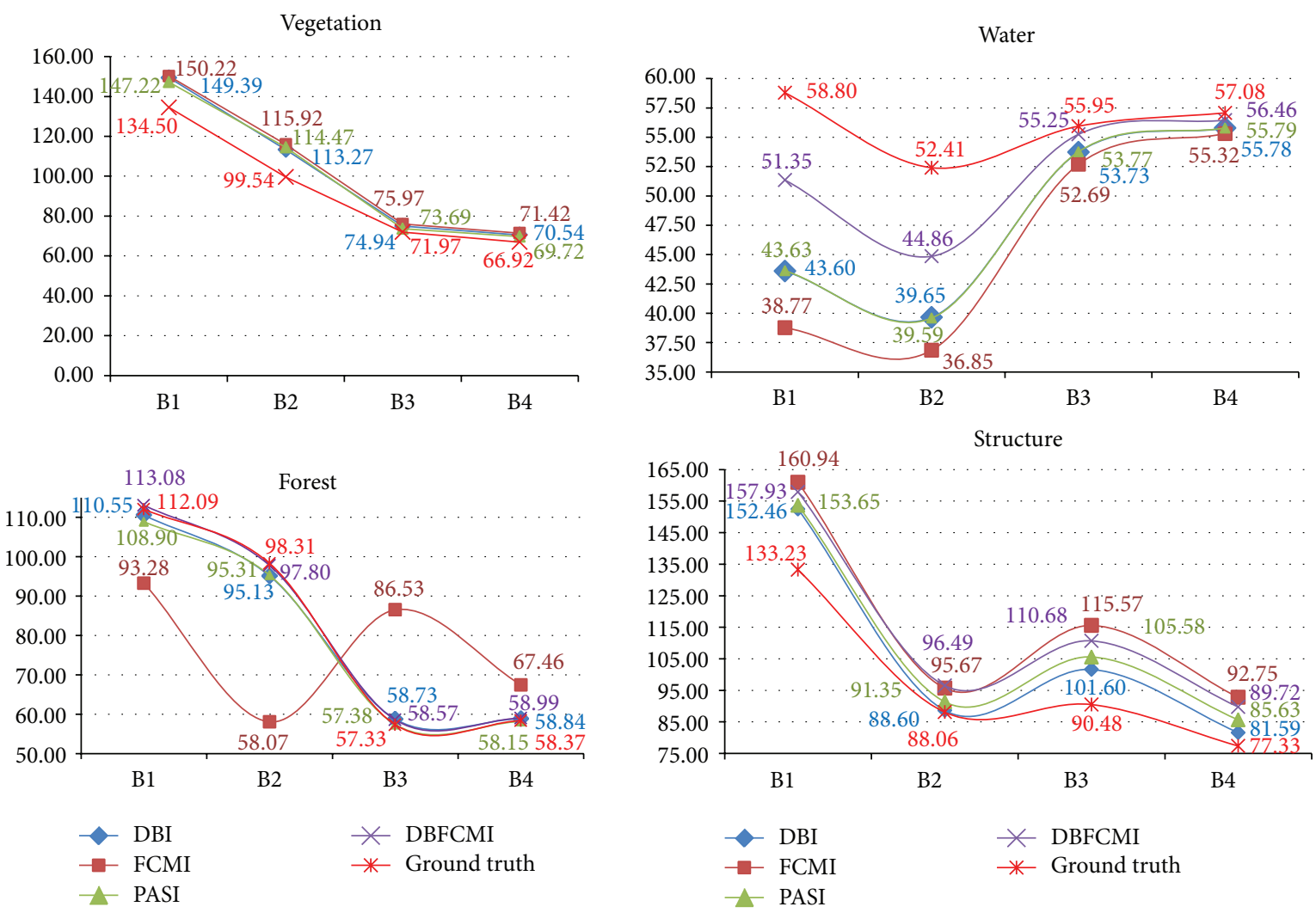

FIGURE 6: Curve comparison of spectral centers between ground truth and landuse classified based on optimal solution of four indices.

TABLE 7: Overall accuracy value of each model varying with crossover ways.

\begin{tabular}{lccccc}
\hline \multirow{2}{*}{$\begin{array}{l}\text { Crossover } \\
\text { way }\end{array}$} & \multicolumn{5}{c}{ Index } \\
& DBI & FCMI & PASI & DBFCMI & Average \\
\hline P1 & 74.3 & 71.6 & 72.9 & 74.6 & 73.3 \\
P2 & 71.6 & 73.2 & 64.9 & 75.5 & 71.3 \\
P3 & 70.8 & 72.5 & 71.5 & 72.5 & 71.8 \\
P4 & 68.8 & 74.5 & 68.9 & 74.7 & 71.7 \\
P5 & 70.5 & 70.2 & 70.9 & 75.0 & 71.6 \\
P6 & 68.7 & 64.2 & 56.0 & 75.0 & 73.3 \\
Standard & 1.2 & 3.0 & 1.4 & 0.2 & - \\
deviation & & & & & \\
\hline
\end{tabular}

${ }^{*}$ String length: 8 , population: 30 , selection way: roulette wheel selection, crossover rate: 0.8 , and mutation rate: 0.003 .

centers and ground truth especially in forest for all bands. It can be observed in Figure 6 that DBFCMI presents its superiority of conformation in the distribution. On the other hand, the results of DBI and PASI can get the optimal solution inferior to DBFCMI; nevertheless, comparing to FCMI, their classified centers are not only closer to ground truth but also with a curve as smooth as the ground truth is.

According to the foregoing analysis, it is worth to notice that rather than classifying the number of landuses accurately
TABLE 8: K-HAT value of each model varying with crossover ways.

\begin{tabular}{lccccc}
\hline $\begin{array}{l}\text { Crossover } \\
\text { way }\end{array}$ & \multicolumn{5}{c}{ Index } \\
& K-HAT & FCMI & PASI & DBFCMI & Average \\
\hline P1 & 0.32 & 0.31 & 0.33 & 0.36 & 0.33 \\
P2 & 0.36 & 0.34 & 0.24 & 0.48 & 0.34 \\
P3 & 0.26 & 0.33 & 0.30 & 0.32 & 0.30 \\
P4 & 0.29 & 0.35 & 0.34 & 0.36 & 0.34 \\
P5 & 0.35 & 0.25 & 0.26 & 0.37 & 0.31 \\
P6 & 0.23 & 0.20 & 0.10 & 0.39 & 0.23 \\
Standard & 0.05 & 0.06 & 0.09 & 0.03 & - \\
deviation & & & & & \\
\hline
\end{tabular}

${ }^{*}$ String length: 8 , population: 30 , selection way: roulette wheel selection, crossover rate: 0.8 , and mutation rate: 0.003 .

but inconformity with the distribution, the ability of distribution determination possesses the crucial influence upon the optimal solution.

Figures 7 and 8 are the curve comparison of overall accuracy and K-HAT based on four indices varying with the different GA operations, respectively. It can be observed the higher values and stability of overall accuracy and K-HAT are presented, while DBFCMI is adopted and then DBI, FCMI, and PASI are in sequence. FCMI and PASI are based on the fuzzy theory and the curves of their accuracy related to the 


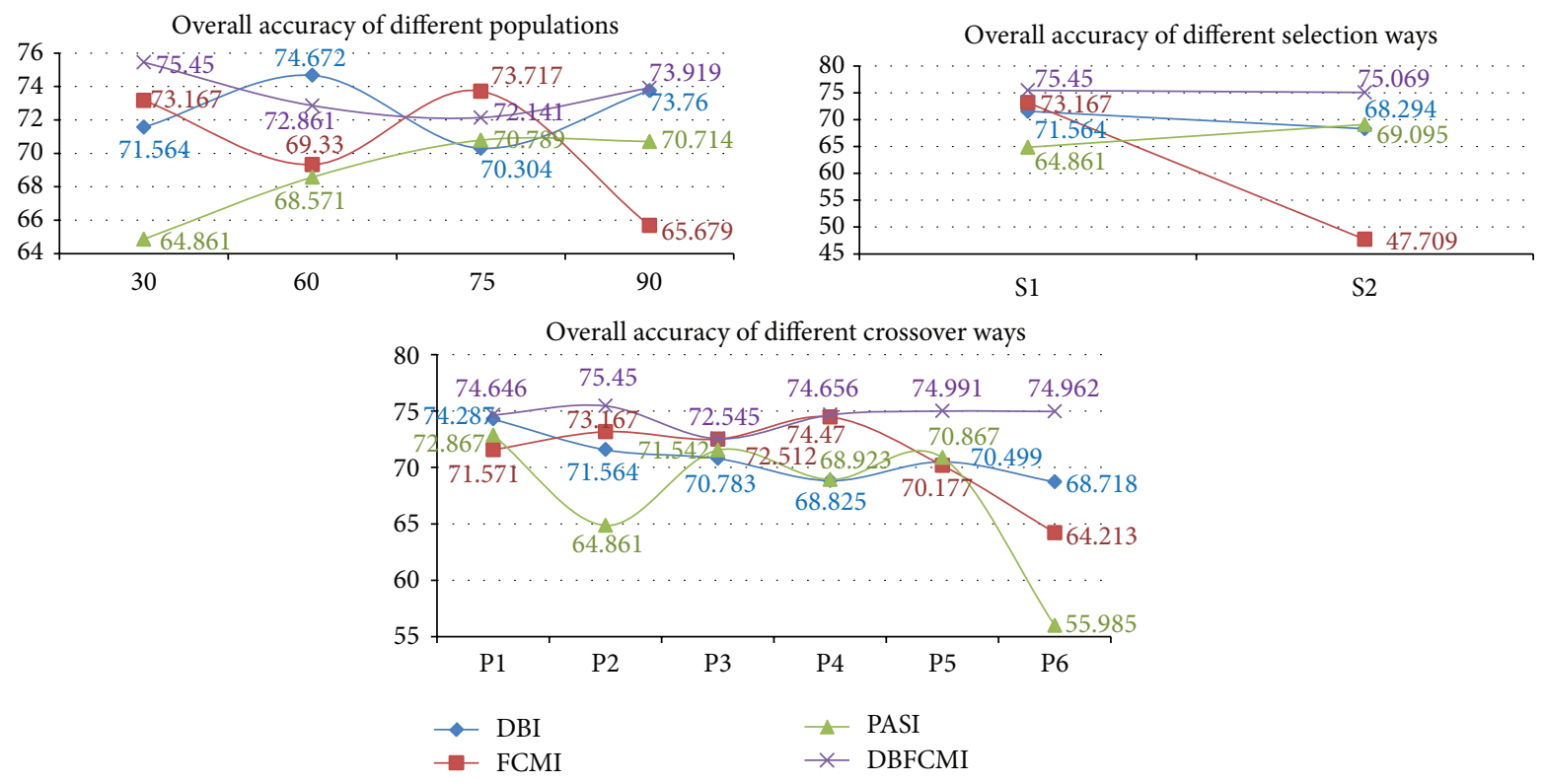

FIGURE 7: Curve comparison of overall accuracy based on four indices varying with different GA operations.
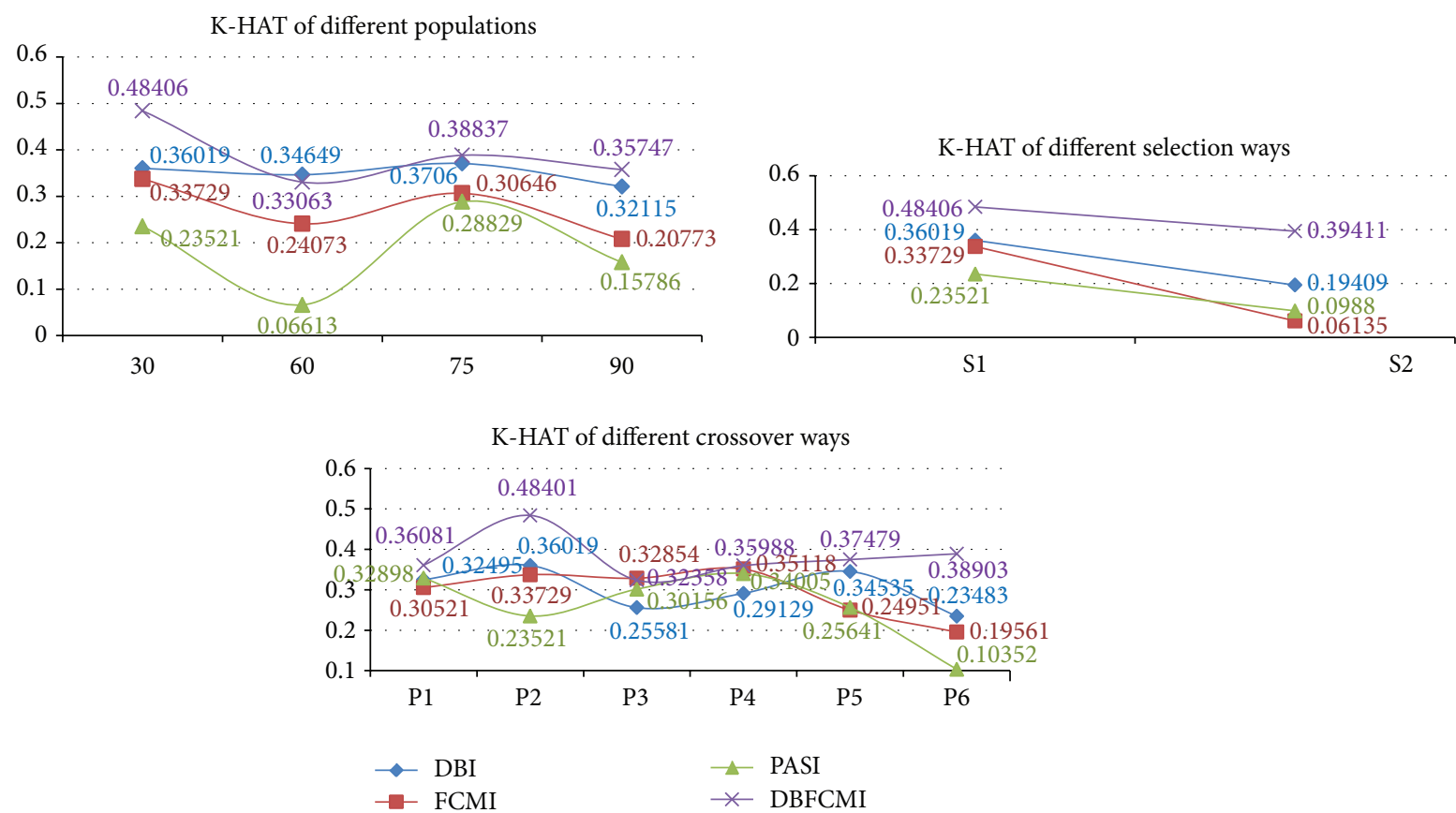

Figure 8: Curve comparison of K-HAT based on four indices varying with different GA operation.

different GA operations vibrate violently rather than a curve as smooth as DBFCMI and DBI. It is remarkable that the overall accuracy and K-HAT of PASI are inferior to the other indices mostly in our test.

Figure 9 is the standard deviation of overall accuracy and K-HAT. It presents the comparison curves of four indices varying with the different GA operations. In the figure, the smallest difference between the different GA operations is presented still at the index of DBFCMI in both overall accuracy and K-HAT. And then DBI, PASI, and FCMI are in sequence. Although four indices are varying with the different selections, FCMI has the largest difference between the four indices varying with the other GA operations. This means that the unstable analyzed results are presented at FCMI more possible than the other indices.

\section{Conclusion}

This paper presented a novel fitness index, DBFCMI, in GA process for the unsupervised classification of SPOT-5 satellite 

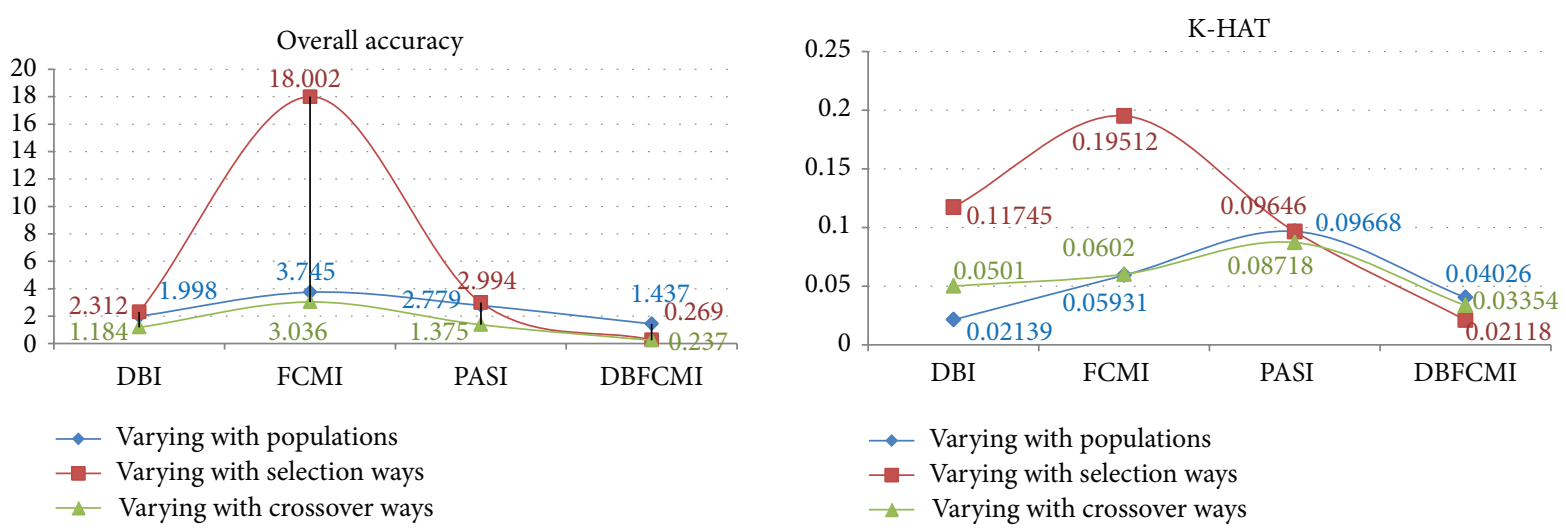

FIGURE 9: Standard deviation comparisons of overall accuracy and K-HAT between the four indices varying with different GA operations.

image. For comparison, three indices, including DaviesBouldin index (DBI), fuzzy C-means index (FCMI), and partition separation index (PASI), were also adopted in GA classification. The conclusion is drawn as follows.

(i) Spectra of bare land and vegetation are as similar as forest in the tested image, so that it is difficult to discriminate the three landuses from each other with GA classifier. Therefore, in most conditions the best associated model of GA can only distinguish bare land and vegetation into forest.

(ii) Overall accuracy and K-HAT are stronger related to distribution of classified landuse than the number of classifications. Besides, except distribution, another critical influence is depending upon the area of landuse especially the landuse with a large area. The best overall accuracy of $75.5 \%$ and the best KHAT of 0.48 were acquired by DBFCMI, with merely three landuses, including forest, water, and structure. However, except the distribution of water and structure which can be determined more identical than the other indices, the largest region of forest can be determined appropriately by DBFCMI as well. Therefore, the influence is not so critical even though the spectra of bare land and vegetation are too similar to forest to be distinguished.

(iii) Comparing with the three indices including DBI, FCMI, and PASI, FCMI and PASI are both based on fuzzy theory so that all the other cluster centers will be considered to influence each independent pixel more or less according to the distance between the pixel and the centers. On the contrary, DBI index based on the classic set theory identifies each pixel in the training data into only one cluster that reduces computation time but results in moderate accuracy. Basically, the physical phenomenon of the spectrum reflection resulted from the neighborhood objects is inevitable. However, sometimes the ideal performances of image classification are obtained by GA coupled with DBI rather than FCMI or PASI. DBFCMI has possessed both advantages of DBI and fuzzy theory and all the examination of this research had been demonstrated that it is effective in the unsupervised image classification. As a result, the best overall accuracy of DBFCMI, DBI, FCMI, and PASI is $75.5 \%, 75.0 \%, 74.9 \%$, and $74.2 \%$ separately. DBFCMI presents $0.75 \%$ increment in the average of the other indices. Overall accuracy is promoted about 1.01\% in average. On the other hand, the best K-HAT of DBFCMI, DBI, FCMI, and PASI is 0.48, 0.37, 0.39, and 0.39 separately. DBFCMI presents 0.1 increments in the average of the other indices. Accordingly, DBFCMI can almost promote K-HAT value to $26.13 \%$ in average.

\author{
Abbreviations \\ $x_{n}$ : $\quad$ Pixel $n$ with grey values $x$ \\ $n$ : Total number of pixels \\ $K: \quad$ Total number of clusters \\ $k_{n}$ : The membership value of $n$th pixel (if the pixel \\ $n$ belongs to $k, k_{n}=1$; otherwise, $k_{n}=1$ ) \\ $v_{k}$ : The centroid of $k$ th cluster \\ $M_{k}$ : The number of pixels belonging to the $k$ th \\ cluster \\ $S_{k}$ : Standard deviation of the pixels in the $k$ th \\ cluster \\ $d_{k j, t}$ : Minkowski distance of order $t$ between the $k$ th \\ and $j$ th centroids. (here, number 2 is chosen for \\ t) \\ $i$ : The $i$ th chromosome \\ $m$ : Total chromosomes of each generation \\ $\mu_{j i}$ : Membership function of pixel $x_{i}$ belonging to \\ the $j$ th cluster \\ $c_{j}: \quad$ Total pixels of $j$ th cluster \\ $\bar{v}$ : The mean of all of the cluster centers.
}

\section{Conflict of Interests}

The authors declare that there is no conflict of interests regarding the publication of this paper. 


\section{References}

[1] T. E. Avery and G. L. Berlin, Fundamentals of Remote Sensing and Airphoto Interpretation, MacMillan Publishing Company, New York, NY, USA, 1992.

[2] T. C. Su, M. D. Yang, T. C. Wu, and J. Y. Lin, "Morphological segmentation based on edge detection for sewer pipe defects on CCTV images," Expert Systems with Applications, vol. 38, no. 10, pp. 13094-13114, 2011.

[3] M. D. Yang, "A genetic algorithm (GA) based automated classifier for remote sensing imagery," Canadian Journal of Remote Sensing, vol. 33, no. 1-4, pp. 203-213, 2007.

[4] M. D. Yang and T. C. Su, "Segmenting ideal morphologies of sewer pipe defects on CCTV images for automated diagnosis," Expert Systems with Applications, vol. 36, no. 2, pp. 3562-3573, 2009.

[5] M. D. Yang and T. C. Su, "Automated diagnosis of sewer pipe defects based on machine learning approaches," Expert Systems with Applications, vol. 35, no. 3, pp. 1327-1337, 2008.

[6] M. D. Yang, Y. F. Yang, and S. C. Hsu, "Application of remotely sensed data to the assessment of terrain factors affecting the Tsao-Ling landslide," Canadian Journal of Remote Sensing, vol. 30, no. 4, pp. 593-603, 2004.

[7] T. M. Lillesand, R. W. Kiefer, and J. W. Chipman, Remote Sensing and Image Interpretation, John Wiley \& Sons, New York, NY, USA, 6th edition, 2007.

[8] J. Tang, L. Wang, and Z. Yao, "Spatio-temporal urban landscape change analysis using the Markov chain model and a modified genetic algorithm," International Journal of Remote Sensing, vol. 28, no. 15, pp. 3255-3271, 2007.

[9] X. Tong, X. Zhang, and M. Liu, "Detection of urban sprawl using a genetic algorithm-evolved artificial neural network classification in remote sensing: a case study in Jiading and Putuo districts of Shanghai, China," International Journal of Remote Sensing, vol. 31, no. 6, pp. 1485-1504, 2010.

[10] M. M. Awad and K. Chehdi, "Satellite image segmentation using hybrid variable genetic algorithm," International Journal of Imaging Systems and Technology, vol. 19, no. 3, pp. 199-207, 2009.

[11] A. Jubai, B. Jing, and J. Yang, "Combining fuzzy theory and a genetic algorithm for satellite image edge detection," International Journal of Remote Sensing, vol. 27, no. 14, pp. 3013-3024, 2006.

[12] C. J. Zhang and X. D. Wang, "Typhoon cloud image enhancement and reducing speckle with genetic algorithm in stationary wavelet domain," IET Image Processing, vol. 3, no. 4, pp. 200-216, 2009.

[13] M. D. Yang and T. C. Su, "An optimization model of sewage rehabilitation," Journal of the Chinese Institute of Engineers, vol. 30, no. 4, pp. 651-659, 2007.

[14] M. D. Yang and T. C. Su, "Automation model of sewerage rehabilitation planning," Water Science and Technology, vol. 54, no. 11-12, pp. 225-232, 2006.

[15] S. Bandyopadhyay and U. Maulik, "Genetic clustering for automatic evolution of clusters and application to image classification," Pattern Recognition, vol. 35, no. 6, pp. 1197-1208, 2002.

[16] S. Bandyopadhyay and U. Maulik, "An evolutionary technique based on K-Means algorithm for optimal clustering in $\mathrm{R}^{N}$," Information Sciences, vol. 146, no. 1-4, pp. 221-237, 2002.

[17] M. S. Yang and K. L. Wu, "A new validity index for fuzzy clustering," in Proceedings of the 10th IEEE International Conference on Fuzzy Systems, pp. 89-92, December 2001.
[18] Y. Bazi, F. Melgani, L. Bruzzone, and G. Vernazza, "A genetic expectation-maximization method for unsupervised change detection in multitemporal SAR imagery," International Journal of Remote Sensing, vol. 30, no. 24, pp. 6591-6610, 2009.

[19] J. C. Bezdek, Pattern Recognition with Fuzzy Objective Function Algorithms, Plenum Press, New York, NY, USA, 1981.

[20] D. A. Coley, An Introduction to Genetic Algorithms for Scientists and Engineers, World Scientific Publishing, Hackensack, NJ, USA, 1999.

[21] Z. Liu, A. Liu, C. Wang, and Z. Niu, "Evolving neural network using real coded genetic algorithm (GA) for multispectral image classification," Future Generation Computer Systems, vol. 20, no. 7, pp. 1119-1129, 2004.

[22] S. N. Sivanandam and S. N. Deepa, Introduction to Genetic Algorithms, Springer, Berlin, Germany, 2008.

[23] A. K. Maiti and M. Maiti, "Discounted multi-item inventory model via genetic algorithm with Roulette wheel selection, arithmetic crossover and uniform mutation in constraints bounded domains," International Journal of Computer Mathematics, vol. 85, no. 9, pp. 1341-1353, 2008.

[24] J. C. Dunn, "A fuzzy relative of the ISODATA process and its in detecting compact well-separated clusters," Journal of Cybernetics, vol. 3, no. 3, pp. 32-57, 1974.

[25] J. T. Kuo, W. S. Lung, C. P. Yang, W. C. Liu, M. D. Yang, and T. S. Tang, "Eutrophication modelling of reservoirs in Taiwan," Environmental Modelling and Software, vol. 21, no. 6, pp. 829844, 2006.

[26] M. D. Yang, J. Y. Lin, C. Y. Yao, J. Y. Chen, T.-C. Su, and C. D. Jan, "Landslide-induced levee failure by high concentrated sediment flow-a case of Shan-An levee at Chenyulan River, Taiwan," Engineering Geology, vol. 123, no. 1-2, pp. 91-99, 2011.

[27] J. Y. Lin, M. D. Yang, B. R. Lin, and P. S. Lin, "Risk assessment of debris flows in Songhe Stream, Taiwan," Engineering Geology, vol. 123, no. 1-2, pp. 100-112, 2011.

[28] M. D. Yang, T. C. Su, C. H. Hsu, K. C. Chang, and A. M. Wu, "Mapping of the 26 December 2004 tsunami disaster by using FORMOSAT-2 images," International Journal of Remote Sensing, vol. 28, no. 13-14, pp. 3071-3091, 2007.

[29] Y. F. Yang, P. Lohmann, and C. Heipke, "Genetic algorithms for the unsupervised classification of satellite images," in Proceedings of the Symposium of ISPRS Commission III, Photogrammetric Computer Vision (PCV'06), vol. 36, pp. 179-184, 2006.

[30] C. C. Chen and C. S. Lin, "A GA-based nearly optimal image authentication approach," International Journal of Innovative Computing, Information and Control, vol. 3, no. 3, pp. 631-640, 2007.

[31] J. B. Kim and H. J. Kim, "GA-based image restoration by isophote constraint optimization," EURASIP Journal on Advances in Signal Processing, vol. 2003, no. 3, Article ID 349873, pp. 238-243, 2003. 

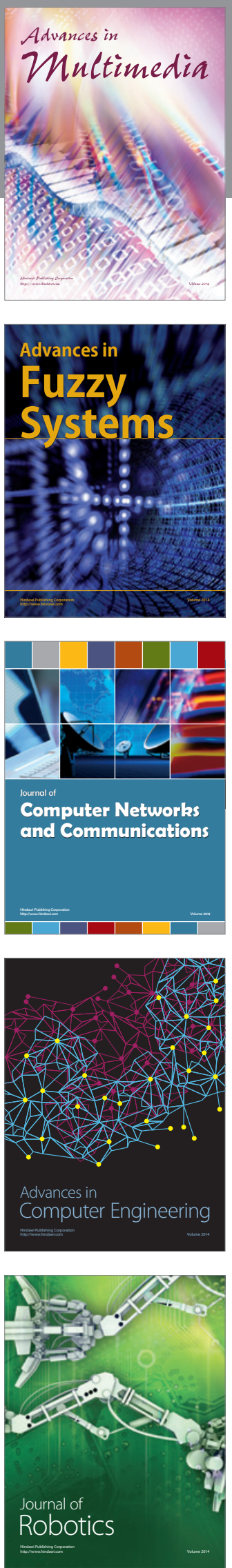

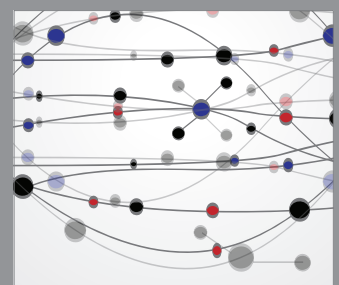

The Scientific World Journal
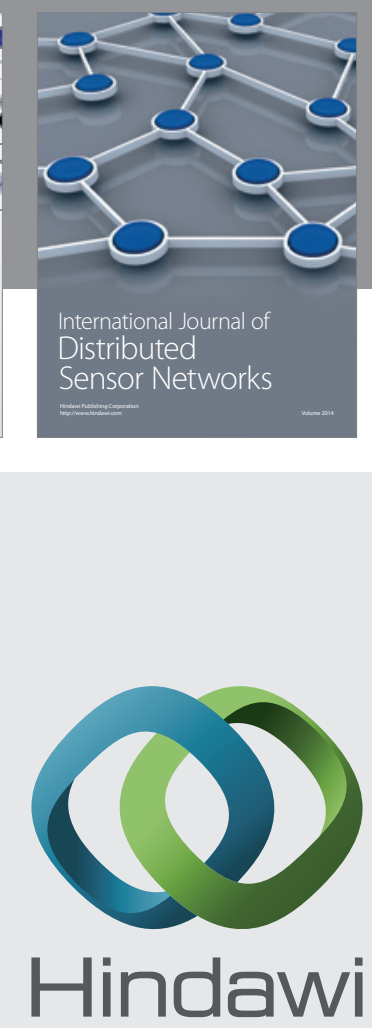

Submit your manuscripts at

http://www.hindawi.com
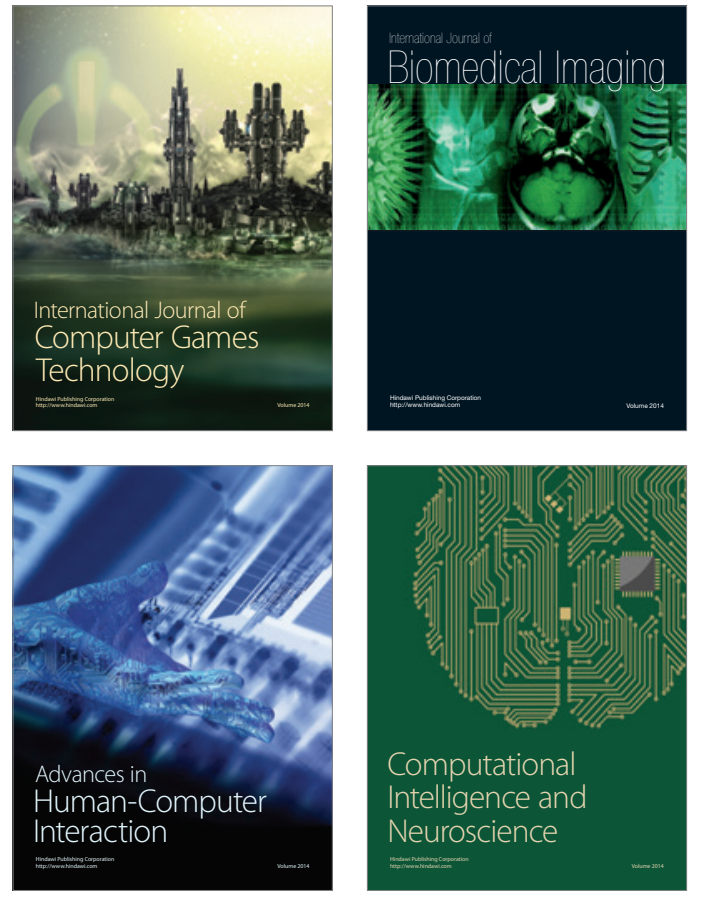
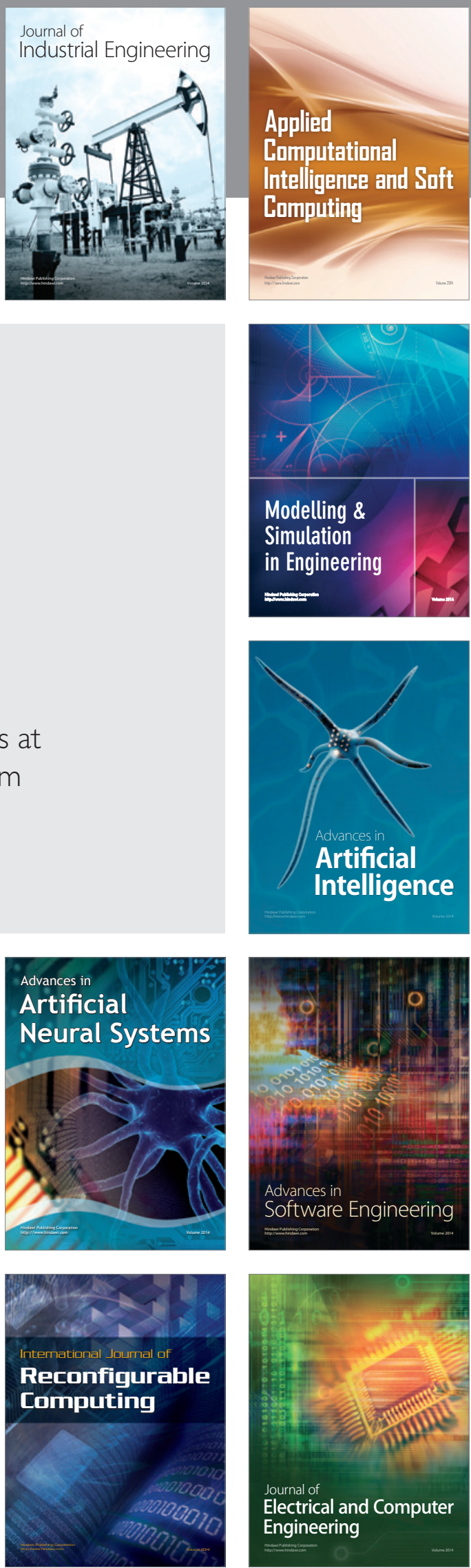